\title{
EEES, TIC y planificación docente: un caso práctico en la Escuela de Ingeniería Informática de la ULPGC
}

\author{
Carmelo Cuenca Hernández \\ Universidad de Las Palmas de Gran Canaria \\ ccuenca@dis.ulpgc.es \\ Francisca Quintana DomínGUEZ \\ Universidad de Las Palmas de Gran Canaria \\ fquintana@dis.ulpgc.es
}

\begin{abstract}
Resumen:
Este trabajo describe cómo afrontar la planificación y puesta en marcha de una asignatura en el marco del EEES. En ese contexto es necesario diseñar actividades que cubran las horas presenciales y no presenciales de la asignatura, así como una forma de tomar evidencias de la realización de las mismas por parte del estudiante, ya que todas las actividades han ser valoradas de cara a realizar la evaluación. Asimismo se presentan los resultados de los tres años en los que la asignatura se ha venido impartiendo siguiendo la planificación propuesta. En general, la planificación de la asignatura ha tenido una buena acogida por parte de los estudiantes que ven cómo el esfuerzo semanal se ve reconocido y no se juegan la asignatura en un examen final. En promedio, más del $88 \%$ de los estudiantes superan la asignatura con esta planificación.
\end{abstract}

Palabras clave: EEES; TIC; planificación docente

\section{EEES, TIC and teaching planning: A practical approach in the Escuela de Ingeniería Informática in the ULPGC}

\begin{abstract}
:
This paper describes how to plan a course in the EEES framework, and how to put it into practice. In this context it is necessary to make a scheduling that includes both attending teaching hours and personal working hours, as well as a way to catch evidences of them, as all of them must contribute to the student's evaluation. It also presents the results of the three years in which the course has been taught following the proposed planning. In general, the planning of the course has been well received by students who perceived that weekly effort is recognized and not played the course in a final exam. On average, over $88 \%$ of the students pass the subject with this planning.
\end{abstract}

Key words: EEES; TIC; teaching planning

\section{Referencia normalizada:}

Cuenca Hernández, C. y Quintana Domínguez, F. (2014): EEES, TIC y planificación docente: un caso práctico en la Escuela de Ingeniería Informática de la ULPGC. Historia y Comunicación Social. Vol. 19. Núm. Especial Enero. Págs. 539-550. 


\section{Sumario:}

1.Introducción 2.¿Cómo se planifica una asignatura dentro del EEES? 2.1.Diseño de las actividades presenciales 2.2.Diseño de las actividades no presenciales 2.3.Planificación temporal 2.4.Evaluación 3.Recursos necesarios para la implantación 4.Resultados de la implantación 5.Conclusiones 6. Agradecimientos 7.Referencias bibliográficas

\section{Introducción}

Las bases del EEES se sentaron con la Declaración de Bolonia (Ministros de Educación de la Unión Europea, 1999), un proyecto impulsado por la Unión Europea para armonizar los sistemas universitarios de los estados miembros. Esta declaración se centraba en el fomento de la cooperación europea para asegurar la calidad en la enseñanza, con el fin de desarrollar criterios y metodologías comparables, la promoción de una necesaria dimensión europea de la educación superior con particular énfasis en el desarrollo curricular y la promoción de la movilidad de los estudiantes y de los profesores de los centros de enseñanza superior europea.

Este nuevo enfoque ha supuesto importantes cambios administrativos, estructurales y metodológicos. Se ha establecido una formación basada en competencias y un sistema de créditos europeos (ECTS) basado en el trabajo del estudiante. Según este modelo, el trabajo anual del estudiante se establece en 1500 horas, que se obtienen de un total de 60 créditos ECTS, cada uno formado por 25 horas de trabajo. Por otro lado, la formación basada en competencias implica un cambio importante, con una metodología de enseñanza-aprendizaje centrada en el estudiante como impulsor de su propia formación, en lugar de ser meros receptores del conocimiento. Los profesores se convierten en instrumentos del proceso de enseñanza-aprendizaje en los que los estudiantes se apoyan para conseguir las competencias formativas. Esto implica que el trabajo autónomo del estudiante cobra una especial relevancia y deben valorarse todos los aspectos del proceso de enseñanza-aprendizaje, lo cual implica que la metodología docente debe cambiar sustancialmente con respecto a la metodología tradicional.

\section{2. ¿Cómo se planifica una asignatura dentro del EEES?}

En el curso académico 2010/11 la Escuela de Ingeniería Informática de la Universidad de Las Palmas de Gran Canaria se dispuso a implantar el nuevo plan de estudios de Graduado en Ingeniería Informática (ULPGC, 2011). En aquel momento, los profesores nos enfrentamos al reto de planificar estas nuevas asignaturas siguiendo el nuevo modelo del EEES. En concreto, la asignatura sobre la que trata esta experiencia de campo, denominada Fundamentos de los Computadores, es una asignatura de 6 créditos ECTS y pertenece al módulo de Formación Básica, impartiéndose durante el segundo semestre del primer curso. La asignatura supone para el estudiante un total 
de 150 horas de trabajo, de las cuales 60 son presenciales y 90 son no presenciales. Se hacía necesario pensar en una planificación en la que todas las actividades que el estudiante desarrollara formaran parte de la planificación de horas totales, y contribuyeran y fueran valoradas de cara a conseguir las competencias que la asignatura tiene asignadas, siguiendo también el modelo de formación basado en competencias.

Durante el proceso de planificación nos dimos cuenta enseguida de que con más de 200 estudiantes matriculados en la asignatura y con la gran cantidad de trabajo no presencial que debíamos diseñar, monitorizar y evaluar, debíamos hacer uso de herramientas basadas en las Tecnologías de la Información y las Comunicaciones (TIC). Por un lado estas herramientas permiten al estudiante romper el tiempo y el espacio, en el sentido de que permiten realizar las actividades no presenciales en cualquier momento y lugar, siempre que se tenga disponible un equipo con conexión a Internet. Por otro lado se trata de herramientas muy valiosas para el profesorado, pues facilitan la toma de evidencias, el seguimiento y la evaluación de una gran cantidad de estudiantes.

\subsection{Diseño de las actividades presenciales}

Las actividades presenciales que decidimos incluir en la planificación de la asignatura son de dos tipos, las sesiones teóricas en el aula, en grupo grande de estudiantes, y las sesiones prácticas en el laboratorio, con grupos reducidos de estudiantes.

Las sesiones teóricas consisten en la impartición en el aula de los contenidos más relevantes del bloque temático por parte del profesor, haciendo énfasis en los apartados más importantes. Para hacer estas sesiones fuesen ligeras y dinámicas se decidió encomendar a los estudiantes como requisito el haber realizado una aproximación previa al tema que se tratará en la sesión teórica correspondiente. De este modo el objetivo de estas sesiones no será introducir el tema sino permitir a los estudiantes reforzar contenidos no asimilados en las lecturas, resolver cuestiones y aclarar las lagunas existentes en cuanto a conceptos y procedimientos, realizando intervenciones en este sentido. La consecución de los objetivos se ve reforzada por el uso de material audiovisual de soporte que está disponible con antelación para los estudiantes a través de las herramientas TIC que la Universidad pone a nuestra disposición.

Las sesiones prácticas están pensadas para ser realizadas en el laboratorio utilizando una herramienta de captura y simulación de circuitos (Xilinx Foundation, 2013). En general consisten en la captura y simulación de un circuito acorde a la temática que se esté estudiando en el tema. Cuando el temario ha avanzado lo suficiente, y los estudiantes ya disponen de los conocimientos necesarios, la complejidad de las sesiones prácticas aumenta e incluye también la realización del diseño del circuito que luego se captura y se simula con la herramienta. El libro de prácticas de la asignatura, desarrollado por los profesores expresamente para la misma, constituye un material imprescindible, y también está disponible para los estudiantes a través de la plataforma TIC. 


\subsection{Diseño de las actividades no presenciales}

Las actividades no presenciales de la asignatura constituyen un $60 \%$ del tiempo total y a la hora de diseñarlas pensamos que debían cumplir dos objetivos. Por un lado, deben servir como preparación previa, necesaria para las actividades presenciales, y por otro lado permitir asimilar, reforzar y practicar los contenidos ya impartidos. Recurrimos a la plataforma TIC para recoger evidencias del aprovechamiento que el estudiante realiza de todas estas actividades, lo que nos permite realizar una evaluación más completa de todo el proceso de enseñanza-aprendizaje. Las actividades no presenciales que decidimos incluir en la asignatura fueron lecturas obligatorias, sesiones de ejercicios, sesiones de estudio, cuestionarios de autoevaluación y trabajo de curso académicamente dirigido.

Las lecturas obligatorias permiten realizar una primera aproximación a la temática que se quiere trabajar. Para cada bloque temático se indican unos ítems bibliográficos a leer y asimilar, y se deben realizar siempre como primera actividad de cada nuevo tema. Cada lectura lleva aparejada la realización de un cuestionario que debe contestarse una vez realizada la lectura, de este modo se evalúa el nivel de asimilación de los contenidos leídos. En estos cuestionarios el uso de las TIC es fundamental ya que debe realizarse dentro de unas fechas determinadas y nos permitirá la corrección automática del mismo. Como veremos más adelante, es fundamental la planificación de fechas en las que realizar estos cuestionarios pues permitirán lograr un adecuado progreso en tiempo y forma en el proceso global de enseñanza-aprendizaje. Asegurándonos de que el estudiante ha realizado la lectura, y el cuestionario correspondiente a la misma, antes de la impartición de los contenidos en el aula podemos garantizar que los estudiantes se encuentran en la mejor disposición para poder afrontar una aproximación crítica a los contenidos que se impartirán de forma presencial.

Las sesiones de ejercicios las forman ejercicios prácticos sobre la temática que se esté tratando. Se incluyeron dentro de las actividades a realizar porque nos pareció una actividad fundamental ya que permiten poner en práctica los conocimientos y destrezas adquiridos en cada tema. Al igual que en el caso anterior, las TIC juegan un papel fundamental ya que permiten a los estudiantes el acceso y entrega de las sesiones de ejercicios, y facilitan a los profesores las tareas de evaluación y seguimiento de los estudiantes.

Las sesiones de estudio tienen como objetivo clarificar y fortalecer los conocimientos, técnicas y procedimientos de cada tema de la asignatura. Para cada tema será necesario que el estudiante realice sesiones de estudio que le permitan consolidar los conocimientos del tema.

Los cuestionarios de autoevaluación ponen el colofón a todo el proceso de enseñanza-aprendizaje del estudiante, ya que permite al estudiante autoevaluarse de los conocimientos y procedimientos de cada tema. Aunque originalmente pensamos que fuese sólo una herramienta de evaluación para ser utilizadas sólo a final de cada tema, posteriormente pensamos que estos cuestionarios podrían utilizarse a lo largo de toda 
la asignatura, permitiendo a los estudiantes repasar y practicar cualquier tema en cualquier momento. Para poder hacer esto último se hacía necesario utilizar las TIC como herramienta, diseñando cuestionarios que estén abiertos durante todo el curso y se puedan intentar tantas veces como se quiera. En cada intento realizado se selecciona un subconjunto diferente de preguntas del mismo tipo, de entre las existentes en el repertorio de preguntas de cada tema, desarrolladas por los profesores de la asignatura. Esta actividad permite al estudiante conocer el nivel de asimilación de los contenidos de cada tema, lo que le permite saber en qué ha fallado, qué partes debe volver a repasar y facilita la realimentación que el estudiante necesita para conocer en qué momento ha alcanzado las competencias formativas del tema. El estudiante debe realizar repetidos intentos del cuestionario de autoevaluación de cada tema hasta haber alcanzado una nota que garantice que ha superado con éxito la asimilación y puesta en práctica de los contenidos y procedimientos del tema.

El trabajo de curso académicamente dirigido consiste en realizar el diseño, simulación e implementación de un circuito digital real a partir de una especificación inicial expresada en lenguaje natural, y permite poner en práctica, de forma global, los contenidos de la asignatura. El procedimiento estipulado para el adecuado desarrollo de esta actividad se ha pensado de forma que el estudiante reciba realimentación en varias fases del proceso. Una vez validado el diseño global se realiza una memoria del trabajo donde se expondrán las principales decisiones de diseño adoptadas y se procederá a realizar una entrevista con el profesor para exponer el trabajo realizado.

\subsection{Planificación temporal}

Una vez seleccionadas las actividades presenciales y no presenciales es necesario combinarlas de la forma adecuada para alcanzar los objetivos formativos que se persiguen. Para cada bloque temático incluido en la asignatura el orden cronológico de las actividades debe ser: lectura previa, sesiones teóricas, sesiones de estudio, sesiones de ejercicios, trabajo práctico en el laboratorio y autoevaluación. Un caso aparte lo forma el trabajo de curso académicamente dirigido, que se debe realizar en la segunda mitad de la asignatura, cuando los estudiantes ya tienen unos conocimientos mínimos que les permitan abordar este tipo de problemas con garantías de éxito.

Por lo tanto, debe existir un hilo conductor entre las actividades de lecturas, sesiones teóricas, ejercicios, sesiones prácticas y autoevaluaciones, donde las sesiones de estudio se intercalen de manera que sustenten el proceso completo de aprendizaje y adquisición de competencias. Para cada semana se planifican una serie de tareas a realizar, y dentro de la semana los estudiantes deciden el momento concreto en el que realizar cada actividad. Es necesario aclarar que en una semana tipo se simultanean una lectura (de un tema que se verá la semana siguiente en las sesiones de teoría) y su cuestionario correspondiente, una sesión de ejercicios (que se corresponde con los contenidos que se están viendo esa semana en las sesiones de teoría), una sesión práctica (de una práctica que se está realizando sobre los contenidos que se están viendo o que ya se han visto), posiblemente una lectura de la práctica siguiente (si la siguiente semana comienza una nueva práctica), sesiones de estudio (del tema actual) y estarán 
abiertos los cuestionarios de autoevaluación de los temas ya terminados. En la Tabla 1 se muestra como ejemplo la planificación de tareas no presenciales a realizar en las primeras cuatro semanas de la asignatura, tal cual aparecen en la herramienta TIC Moodle (MOODLE, 2013) que utilizamos para la asignatura.

Tabla 1: Planificación de tareas a realizar a través de la herramienta Moodle durante las primeras semanas.

28 de enero - 3 de febrero

\section{SEMANA 1}

LP1_Lectura_Práctica1 (no se hace test)

Q L1_Algebra de Boole y expresiones lógicas

QL1_Test

D E1_A_Algebra de Boole y expresiones lógicas

$\square$ E1_A Introduce tus respuestas

4 de febrero - 10 de febrero

SEMANA 2

L2_Karnaugh_funciones_incompletas_multiples_ V12_Test

Q E1_B_Algebra de Boole y puertas lógicas

Q1 E1_B_Introduce tus respuestas

Autoevaluación Tema 1

\section{1 de febrero - 17 de febrero}

\section{SEMANA 3}

๑ L3_Componentes combinacionales básicos V] L3_Test

( E2_Simplificación de funciones lógicas Q1 E2_Introduce tus respuestas 1 Cuestionario Práctica 1

\section{8 de febrero - 24 de febrero}

\section{SEMANA 4}

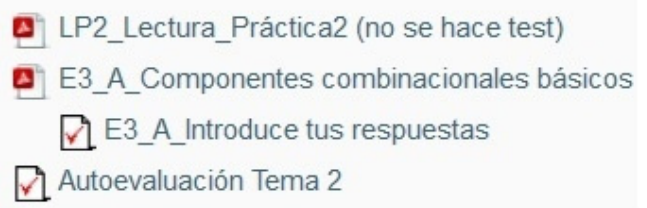

凶 LP2_Lectura_Práctica2 (no se hace test) 口] E3_A_Introduce tus respuestas 7 Autoevaluación Tema 2

En la Tabla 2 se muestra la planificación completa de la asignatura. En esta tabla la nomenclatura utilizada es la siguiente: Tn (sesión de teoría del tema n), Pn (sesión de prácticas en laboratorio de la práctica n), LPn (lectura de la práctica n), Ln (lectura del tema n), Sn (sesión de estudio del tema n), En (sesión de ejercicios del tema n; puede haber varias, en cuyo caso se numeran con letras consecutivas, por ejemplo E1_A, E1_B en la Tabla 2), TC (horas dedicadas al trabajo de curso dirigido).

Cada fila de la Tabla 2 indica la serie de actividades que deben realizar los estudiantes en la semana indicada. Las horas de realización de los diversos test y cuestionarios, que van aparejados con algunas de las actividades, están incluidas dentro de la propia planificación, en el tiempo que se ha fijado para la correspondiente actividad. Consideramos fundamental fijar una ventana temporal para introducir las respuestas de los cuestionarios ya que garantiza que los estudiantes siguen la temporalización establecida dentro de la planificación de la asignatura. 
Tabla 2: Planificación semanal completa de la asignatura.

\begin{tabular}{|c|c|c|c|c|c|c|c|c|c|c|c|c|c|}
\hline \multirow{4}{*}{$\begin{array}{c}\text { Semana } \\
1\end{array}$} & \multicolumn{3}{|c|}{$\begin{array}{c}\text { Presencial } \\
\text { (4 h/semana) }\end{array}$} & \multicolumn{10}{|c|}{$\begin{array}{l}\text { No presencial } \\
\text { (6 h/semana) }\end{array}$} \\
\hline & \multicolumn{2}{|c|}{$\begin{array}{l}\text { Sesión } \\
\text { teórica }\end{array}$} & \multirow{3}{*}{$\begin{array}{c}\begin{array}{c}\text { Sesión } \\
\text { práctica } \\
(\mathbf{2 h})\end{array} \\
\text { P1 }\end{array}$} & \multirow{2}{*}{\multicolumn{2}{|c|}{ Lecturas }} & \multirow{2}{*}{\multicolumn{2}{|c|}{ Autoeval }} & \multirow{2}{*}{\multicolumn{2}{|c|}{$\begin{array}{l}\text { Sesión } \\
\text { Estudio }\end{array}$}} & \multirow{2}{*}{\multicolumn{2}{|c|}{$\begin{array}{c}\text { Sesiones } \\
\text { Ejercicios }\end{array}$}} & \multirow{2}{*}{\multicolumn{2}{|c|}{$\begin{array}{c}\text { Trabajo de } \\
\text { curso } \\
\text { dirigido }\end{array}$}} \\
\hline & $1 \mathrm{~h}$ & $1 \mathrm{~h}$ & & & & & & & & & & & \\
\hline & $\mathrm{T} 1$ & $\mathrm{~T} 1$ & & $\begin{array}{l}\text { LP1 } \\
\text { L1 }\end{array}$ & $\begin{array}{l}1 \mathrm{~h} \\
1 \mathrm{~h}\end{array}$ & - & - & $\mathrm{S} 1$ & $2 \mathrm{~h}$ & E1-A & $2 \mathrm{~h}$ & - & - \\
\hline 2 & $\mathrm{~T} 1$ & $\mathrm{~T} 2$ & $\mathrm{Pl}$ & $\mathrm{L} 2$ & $2 \mathrm{~h}$ & Al & $1 \mathrm{~h}$ & $\mathrm{~S} 2$ & $2 \mathrm{~h}$ & E1-B & $1 \mathrm{~h}$ & - & - \\
\hline 3 & $\mathrm{~T} 2$ & $\mathrm{~T} 2$ & $\mathrm{P} 1$ & L3 & $1 \mathrm{~h}$ & - & - & S3 & $3 \mathrm{~h}$ & E2 & $2 \mathrm{~h}$ & - & - \\
\hline 4 & T3 & $\mathrm{T} 3$ & $\mathrm{P} 2$ & LP2 & $1 \mathrm{~h}$ & $\mathrm{~A} 2$ & $1 \mathrm{~h}$ & $\mathrm{~S} 4$ & $3 \mathrm{~h}$ & E3-A & $1 \mathrm{~h}$ & - & - \\
\hline 5 & $\mathrm{~T} 3$ & $\mathrm{~T} 4$ & $\mathrm{P} 2$ & L4 & $2 \mathrm{~h}$ & - & - & S5 & $2 \mathrm{~h}$ & E3-B & $2 \mathrm{~h}$ & - & - \\
\hline 6 & $\mathrm{~T} 4$ & T5 & P3 & $\begin{array}{l}\text { LP3 } \\
\text { L5 }\end{array}$ & $\begin{array}{l}1 \mathrm{~h} \\
1 \mathrm{~h}\end{array}$ & A3 & $1 \mathrm{~h}$ & S6 & $2 \mathrm{~h}$ & E4 & $1 \mathrm{~h}$ & - & - \\
\hline 7 & $\mathrm{~T} 5$ & T5 & P3 & L6 & $1 \mathrm{~h}$ & A4 & $1 \mathrm{~h}$ & S7 & $3 \mathrm{~h}$ & E5-A & $1 \mathrm{~h}$ & - & - \\
\hline 8 & T6 & $\mathrm{T} 6$ & $\mathrm{P} 4$ & LP4 & $1 \mathrm{~h}$ & - & - & S8 & $2 \mathrm{~h}$ & E5-B & $2 \mathrm{~h}$ & $\mathrm{TC}$ & $1 \mathrm{~h}$ \\
\hline 9 & T6 & $\mathrm{T} 7$ & $\mathrm{P} 4$ & L7 & $\mathrm{lh}$ & A5 & $\mathrm{lh}$ & S9 & $3 \mathrm{~h}$ & E6 & $\mathrm{lh}$ & $\mathrm{TC}$ & lh \\
\hline 10 & $\mathrm{~T} 7$ & $\mathrm{~T} 7$ & P5 & LP5 & $1 \mathrm{~h}$ & A6 & $1 \mathrm{~h}$ & $\mathrm{~S} 10$ & $2 \mathrm{~h}$ & E7 & $1 \mathrm{~h}$ & $\mathrm{TC}$ & $1 \mathrm{~h}$ \\
\hline 11 & $\mathrm{~T} 7$ & $\mathrm{~T} 8$ & P5 & L8A & $1 \mathrm{~h}$ & A7 & $1 \mathrm{~h}$ & $\mathrm{~S} 11$ & $2 \mathrm{~h}$ & E8-A & $1 \mathrm{~h}$ & $\mathrm{TC}$ & $1 \mathrm{~h}$ \\
\hline 12 & $\mathrm{~T} 8$ & $\mathrm{~T} 8$ & P5 & L8B & $1 \mathrm{~h}$ & - & - & $\mathrm{S} 12$ & $2 \mathrm{~h}$ & E8-B & $1 \mathrm{~h}$ & $\mathrm{TC}$ & $2 \mathrm{~h}$ \\
\hline 13 & T8 & T8 & P6 & LP6 & $1 \mathrm{~h}$ & - & - & S13 & $2 \mathrm{~h}$ & E8-C & $1 \mathrm{~h}$ & $\mathrm{TC}$ & $2 \mathrm{~h}$ \\
\hline 14 & $\mathrm{~T} 8$ & T8 & P6 & L9 & $1 \mathrm{~h}$ & A 8 & $1 \mathrm{~h}$ & $\mathrm{~S} 14$ & $2 \mathrm{~h}$ & E8-D & $1 \mathrm{~h}$ & $\mathrm{TC}$ & $1 \mathrm{~h}$ \\
\hline 15 & T9 & T9 & P6 & - & - & A9 & $1 \mathrm{~h}$ & S15 & $4 \mathrm{~h}$ & - & - & TC & $1 \mathrm{~h}$ \\
\hline
\end{tabular}

\subsection{La evaluación}

El modelo EEES recoge que en la evaluación deben valorarse todos los aspectos del proceso de enseñanza-aprendizaje. Para ello se hace uso de herramientas TIC que nos permiten recoger evidencias a través de la realización de cuestionarios, tanto para las actividades presenciales como para las no presenciales. Para la generación de cada cuestionario, diferente para cada estudiante, se selecciona un conjunto de preguntas de entre una batería de preguntas específicas para la actividad a evaluar. Además se recoge una evidencia de participación activa del estudiante en las sesiones presenciales, puntuándose positivamente todas las aportaciones de calidad y enriquecedoras para el grupo. Por otro lado, el trabajo de curso se evalúa con una memoria y una entrevista, valorándose aspectos como la consecución de objetivos, el esfuerzo, manejo de herramientas, espíritu crítico, consolidación de conocimientos y pericia en la puesta en práctica de los contenidos. Finalmente, se realiza un examen de la asignatura, que no es necesario aprobar, pero que contribuye a la nota final del estudiante.

En el cálculo de la nota final (NF) se realiza la media ponderada de las notas de los cuestionarios de las lecturas (NL), las sesiones de ejercicios (NE), las prácticas de laboratorio (NP), el trabajo de curso dirigido (NTC), el examen final (NEF) y la participación activa del estudiante (NPA), según la fórmula:

$$
\mathrm{NF}=0.1 * \mathrm{NL}+0.1 * \mathrm{NE}+0.3 * \mathrm{NP}+0.1 * \mathrm{NTC}+0.35 * \mathrm{NEF}+0.05 * \mathrm{NPA}
$$


Nótese que los cuestionarios de autoevaluación, aunque son obligatorios, no contribuyen con puntuación a la nota final. Esto se debe a que se proporcionan como herramienta de estudio y autovaloración de cada tema, y se exige como condición necesaria el haber realizado todos esos cuestionarios y haber obtenido una nota superior a siete puntos en cada uno de ellos.

\section{Recursos necesarios para la implantación}

La implantación de una planificación como la expuesta en una asignatura con más de 200 estudiantes matriculados requiere obligatoriamente del uso de herramientas automáticas basadas en las TIC, ya que es la única forma de poder recolectar la cantidad de evidencias que hemos planteado. Aun así el esfuerzo a realizar es considerable, ya que se ha planteado la realización de 37 cuestionarios por parte de los estudiantes, y cada uno de ellos debe generarse a partir de una bolsa de preguntas, que debe ser lo suficientemente grande como para que el cuestionario que se genere garantice cierta variabilidad.

Como herramienta TIC se ha utilizado el Moodle, proporcionado por la ULPGC a través del Campus Virtual (CAMPUS VIRTUAL DE LA ULPGC, 2013). Esta plataforma es muy potente y permite proporcionar un repositorio en el que colocar el material audiovisual de apoyo a las sesiones teóricas y prácticas de laboratorio. También permite la posibilidad de crear bases de datos de preguntas organizadas por temas, de las cuales pueden seleccionarse las cuestiones para confeccionar automáticamente los cuestionarios que les serán presentados a los estudiantes para las diferentes actividades. Las sesiones de los ejercicios también se realizan a través del Moodle, introduciendo los resultados de las hojas de ejercicios planteadas para cada tema. En todos los casos se puede seleccionar la ventana temporal en la cual están disponibles dichos cuestionarios. Entre otra funcionalidad, el Moodle también permite organizar a los estudiantes en grupos, comunicarse con los estudiantes de forma grupal o individualizada, así como la realización de tutorías privadas o en grupo a través de la plataforma.

La planificación propuesta requiere también recursos para las prácticas en el laboratorio. En ese sentido se ha desarrollado un libro de prácticas (CUENCA-HERNÁNDEZ, 2013) para la asignatura que estará disponible a través del Moodle de la asignatura. Este libro de prácticas, junto con la herramienta software Xilinx Foundation Series (XILINX FOUNDATION, 2013) garantiza los recursos necesarios para la puesta en marcha de las sesiones de prácticas en laboratorio de la asignatura.

Por último, necesitamos tener en cuenta también los recursos bibliográficos que serán necesarios para poner en marcha la planificación propuesta. Dado que hemos incluido una actividad de lectura obligatoria por semana, debemos garantizar que los estudiantes tienen acceso al material bibliográfico necesario para realizar dicha lectura. En un principio pensamos en realizar las lecturas utilizando un libro actual 
(FLOYD, 2006). Sin embargo, cuando comprobamos que no existía suficiente número de ejemplares en la biblioteca universitaria, y que era inviable adquirir un ejemplar por estudiante, fue preciso ser imaginativos a la hora de encontrar una solución. Dado que los contenidos de la asignatura corresponden a una materia con unos conocimientos sólidos y asentados desde hace bastantes años, escogimos como libro de lecturas un libro clásico (GAJSKI, 2000) que ya está descatalogado en la editorial, motivo por el cual la biblioteca, amparándose en la normativa legal vigente, puede realizar la digitalización del mismo y ofrecerlo on-line a través de la web de la propia biblioteca.

\section{Resultados de la implantación}

La implantación de la planificación expuesta ha tenido una buena acogida por parte de los estudiantes que ven cómo el esfuerzo semanal se ve reconocido y no se juegan la asignatura en un examen final. La desventaja que los estudiantes suelen remarcar es que esta planificación les requiere una dedicación constante a la asignatura, lo que en determinados casos puede suponer una desventaja si el estudiante no se encuentra en disposición de dedicar el número de horas semanales necesarias para llevar la asignatura al día. Esto nos llevó a darnos cuenta de que, aunque en un primer momento consideramos la planificación inamovible e inflexible para todos los estudiantes, existen ciertos casos particulares que es preciso tratar con flexibilidad, ya que están contemplados dentro de la normativa vigente de la Universidad (ULPGC, 2010).

La Tabla 3 muestra los resultados de la asignatura en los tres cursos académicos impartidos. Para cada curso académico se muestra el número de matriculados, el número de estudiantes que han comenzado la asignatura, el número de estudiantes que han realizado la evaluación continua hasta el final (en datos absolutos y porcentuales) y el número de estudiantes que han aprobado la asignatura siguiendo evaluación continua (en datos absolutos y porcentuales). Se muestra también el valor acumulado de los tres cursos como forma de evaluar el comportamiento global.

Tabla 3: Resultados de la asignatura en la evaluación continua

\begin{tabular}{|c|c|c|c|c|c|c|}
\hline \multirow[t]{2}{*}{ Curso } & \multirow{2}{*}{$\begin{array}{l}\quad \mathrm{N}^{\circ} \\
\text { estudiantes } \\
\text { matriculados }\end{array}$} & \multirow{2}{*}{$\begin{array}{l}\mathrm{N}^{\circ} \text { estudiantes } \\
\text { que comenzaron } \\
\text { la asignatura }\end{array}$} & \multicolumn{2}{|c|}{$\begin{array}{c}\text { Estudiantes que finalizaron } \\
\text { la asignatura }\end{array}$} & \multicolumn{2}{|c|}{ Estudiantes aprobados } \\
\hline & & & $\mathrm{N}^{\mathrm{o}}$ & $\%$ & $\mathrm{~N}^{\mathrm{o}}$ & $\%$ \\
\hline $10 / 11$ & 200 & 110 & 56 & $\begin{array}{c}51 \% \\
(56 / 110)\end{array}$ & 50 & $\begin{array}{l}89,3 \% \\
(50 / 56) \\
\end{array}$ \\
\hline $11 / 12$ & 248 & 161 & 94 & $\begin{array}{c}58,4 \% \\
(94 / 161) \\
\end{array}$ & 88 & $\begin{array}{l}93,6 \% \\
(88 / 94) \\
\end{array}$ \\
\hline $12 / 13$ & 221 & 199 & 93 & $\begin{array}{c}46,7 \% \\
(93 / 199) \\
\end{array}$ & 78 & $\begin{array}{l}83,7 \% \\
(78 / 93) \\
\end{array}$ \\
\hline Total & 669 & 470 & 243 & $\begin{array}{c}51,7 \% \\
(243 / 470)\end{array}$ & 216 & $\begin{array}{c}88,8 \% \\
(216 / 243)\end{array}$ \\
\hline
\end{tabular}


El primer aspecto que llama la atención al analizar la Tabla 3 es que el número de estudiantes que comienzan la asignatura es bajo, con respecto al número de estudiantes matriculados, lo que significa que han abandonado la asignatura aún antes de empezarla. Esto puede deberse a que los estudiantes no superan la asignatura que es prerrequisito y no se ven preparados para afrontar el seguimiento de la misma. También puede deberse a que los estudiantes, desde el principio, deciden no optar por la evaluación continua sino por la tradicional evaluación al final. Vemos, sin embargo que esta tendencia ha ido disminuyendo a medida que han pasado los años.

Aunque a los estudiantes se les da la posibilidad de hacer evaluación no continua, en promedio un $51,7 \%$ de los estudiantes prefieren la evaluación continua, según muestran los datos de la Tabla 3, siendo el porcentaje de aprobados superior en promedio al $88 \%$ en los tres cursos en que se ha venido impartiendo. Cabe destacar el curso 2011/2012 donde aprobaron más del 93\% de los estudiantes que siguieron la evaluación continua.

Los estudiantes que decidieron acogerse a la evaluación no continua constituyen un $48,3 \%$ en promedio, y sus resultados, que se muestran en la Tabla 4 , son mucho peores, a pesar de que el examen final es el mismo para ambos grupos. En esta tabla puede apreciarse que las tasas de aprobados en la convocatoria ordinaria oscilan entre el $4 \%$ y el $12 \%$, con un valor promedio de $7,2 \%$. La Tabla 4 también muestra los resultados de la convocatoria extraordinaria donde los porcentajes de aprobados vuelven a tomar valores bastante bajos. En este grupo de estudiantes se encuentran aquellos que no superaron la asignatura en la convocatoria ordinaria, ya fuesen de evaluación continua o no continua. Estos resultados demuestran que la evaluación continua produce mejores resultados que la evaluación puntual al final del semestre.

Tabla 4: Resultados de la asignatura en la evaluación no continua

\begin{tabular}{|l|c|c|c|c|}
\hline \multirow{2}{*}{ Curso } & \multicolumn{2}{|c|}{ Convocatoria Ordinaria } & \multicolumn{2}{c|}{ Convocatoria Extraordinaria } \\
\cline { 2 - 5 } & Presentados & Aprobados & Presentados & Aprobados \\
\hline $2010 / 2011$ & 50 & 2 & 21 & 3 \\
& & $4 \%$ & & $14,3 \%$ \\
\hline $2011 / 2012$ & 25 & 3 & 22 & 1 \\
& & $12 \%$ & & $4,5 \%$ \\
\hline $2012 / 2013$ & 22 & 2 & 22 & 10 \\
& & $9,1 \%$ & & $45,4 \%$ \\
\hline Acumulado & 97 & $7,2 \%$ & 65 & 14 \\
& & & & $21,5 \%$ \\
\hline
\end{tabular}




\section{Conclusiones}

En este trabajo se ha presentado la forma de realizar la planificación de una asignatura dentro del EEES. En este nuevo modelo de enseñanza es preciso diseñar actividades tanto para las horas presenciales como para las no presenciales de trabajo personal del estudiante. Además es preciso diseñar una metodología que permita recoger evidencias de todas las actividades formativas realizadas, ya que el trabajo autónomo cobra una especial relevancia y debe fomentarse, valorarse y tenerse en cuenta en la planificación global. Es importante realizar una planificación con diversas actividades que, sincronizadas adecuadamente, permitan conseguir las competencias formativas de la asignatura.

Los resultados obtenidos muestran que el \% de aprobados entre los estudiantes que realizan la evaluación continua es mucho mayor que entre los que no la realizan, aun siendo evaluados con el mismo examen final. Por este motivo creemos que la evaluación debería realizarse únicamente de forma continua, aunque somos conscientes del rechazo que esta idea puede suscitar entre los estudiantes.

En nuestra opinión, la nueva metodología, basada en el esfuerzo del estudiante, les capacita para formarse de manera integral y para ser proactivos en la adquisición de las competencias formativas, lo que les permite estar mejor preparados para posicionarse en el mercado laboral.

\section{Agradecimientos}

A los autores les gustaría agradecer a los profesores D. Luis Doreste Blanco, D. Sunil Lalchand Khemchadani, D. Ricardo Pérez García y D. Oliverio Jesús Santana Jaria, que han impartido docencia en la asignatura de Fundamentos de los Computadores del Grado en Ingeniería Informática, el esfuerzo realizado en la implantación de la asignatura y la buena acogida y predisposición en la planificación de la misma a lo largo de estos años.

\section{Bibliografía}

\subsection{Libros}

CUENCA-HERNÁNDEZ, C.; QUINTANA-DOMÍNGUEZ, F. (2013). Prácticas de Fundamentos de los Computadores. Las Palmas de Gran Canaria: Universidad de Las Palmas de Gran Canaria

FLOYD, T. (2006). Fundamentos de Sistemas Digitales. Novena Edición. Madrid: Pearson Educación

GAJSKI, D. (2000). Principios de Diseño Digital. Madrid: Prentice Hall 


\subsection{Publicaciones web}

CAMPUS VIRTUAL DE LA ULPGC (2013). Portal web del Campus Virtual de la ULPGC. En: http://www.campusvirtual.ulpgc.es. [28-10-2013].

GOBIERNO DE ESPAÑA (2007). REAL DECRETO 1393/2007, de 29 de octubre, por el que se establece la ordenación de las enseñanzas universitarias oficiales. En Boletín Oficial del Estado $\mathrm{n}^{\circ} 260$ del martes 30 de octubre de 2007. http:// www.boe.es/boe/dias/2007/10/30/pdfs/A44037-44048.pdf. [28-10-2013].

MINISTROS DE EDUCACIÓN DE LA UNIÓN EUROPEA (1999). The Bologna Declaration of 19 June 1999. http://www.eees.es/pdf/Declaracion_Bolonia.pdf [28-10-2013].

MOODLE (2013). Portal web de Moodle. En: http://moodle.org. [28-10-2013].

ULPGC (2010): Acuerdo del Consejo de Gobierno de la Universidad de Las Palmas de Gran Canaria, de 2 de julio de 2010, por el que se aprueba el Reglamento de Planificación Académica de la Universidad de Las Palmas de Gran Canaria. En Boletín Oficial de la Universidad de Las Palmas de Gran Canaria, año 3 , $\mathrm{n}^{\mathrm{o}} 7$, del viernes 2 de julio de 2010. https://www.ulpgc.es/hege/almacen/download/7080/7080401/boulpgc_2_de_julio_2010_.pdf. [28-10-2013].

ULPGC (2011): Resolución de 29 de septiembre de 2011, de la Universidad de Las Palmas de Gran Canaria, por la que se publica el plan de estudios de Graduado en Ingeniería Informática. En Boletín Oficial del Estado $n^{\circ} 248$ del viernes 14 de octubre de 2011. http://boe.es/boe/dias/2011/10/14/pdfs/BOE-A-2011-16170.pdf. [28-10-2013].

XILINX FOUNDATION (2013): Portal web de Xilinx Foundations Series. http:// www.xilinx.com. [28-10-2013].

\section{Los autores}

Carmelo Cuenca Hernández es Licenciado en Ciencias Físicas por la Universidad Complutense de Madrid y Doctor en Informática por la Universidad de Las Palmas de Gran Canaria. Actualmente ocupa una plaza de Titular de Escuela Universitaria adscrita al Departamento de Informática y Sistemas de la Universidad de Las Palmas de Gran Canaria, donde ha estado 24 años impartiendo docencia en la Escuela de Ingeniería Informática en asignaturas del ámbito de la arquitectura y tecnología de los computadores.

Francisca Quintana Domínguez es Licenciada en Informática y Doctora en Informática por la Universidad de Las Palmas de Gran Canaria (ULPGC). Actualmente ocupa una plaza de profesora Titular de Universidad que está adscrita al Departamento de Informática y Sistemas de la ULPGC, donde ha estado 21 años impartiendo docencia en la Escuela de Ingeniería Informática en asignaturas del ámbito de la arquitectura y tecnología de los computadores. 